\title{
Maser and thermal methanol emission in the millimeter wave range: new masers at $1.3 \mathrm{~mm}$ and $2.8 \mathrm{~mm}$
}

\author{
Sergei V. Kalenskii, Vyacheslav I. Slysh, and Irina E. Val'tts \\ Astro Space Center, Lebedev's Physical Institute, Profsoyuznaya str. \\ 84/32, 117810 Moscow, Russia
}

\begin{abstract}
Results of a survey of Galactic star-forming regions in the lines of methanol $8_{-1}-7_{0} E$ at $229.8 \mathrm{GHz}, 3_{-2}-4_{-1} E$ at $230.0 \mathrm{GHz}$, $0_{0}-1_{-1} E$ at $108.9 \mathrm{GHz}$, and a series of methanol lines $J_{1}-J_{0} E$ near $165 \mathrm{GHz}$ are presented. Two masers, DR $21(\mathrm{OH})$ and DR 21 West, and two maser candidates, L 379 IRS3 and NGC $6334 \mathrm{I}(\mathrm{N})$, as well as 16 thermal sources are found at $229.8 \mathrm{GHz}$. This is the first detection of methanol masers at a wavelength as short as $1 \mathrm{~mm}$. At $108.9 \mathrm{GHz}$, masers were found towards G345.01+1.79 and probably, towards M 8E. Thermal emission is found towards 28 objects. Only thermal emission was found at 165 and $230.0 \mathrm{GHz}$ (20 and 7 sources, respectively). The masers at $229.8 \mathrm{GHz}$ belong to class I, whereas those at $108.9 \mathrm{GHz}$ belong to class II, according to the classification by Menten (1991). The masers in DR $21(\mathrm{OH})$ and DR 21 West can be roughly fitted by models with the gas kinetic temperature of the order of $50 \mathrm{~K}$. The detection of the $108.9 \mathrm{GHz}$ masers towards $\mathrm{G} 345.01+1.79$ and $\mathrm{M} 8 \mathrm{E}$ may indicate on a specific geometry of these objects. The combination of the existence of the class II $J_{0}-J_{-1} E$ masers towards W $3(\mathrm{OH}), \mathrm{G} 345.01+1.79$, W 48 , and Cep $\mathrm{A}$ and our non-detection of the $3_{-2}-4_{-1} E$ and $J_{1}-J_{0} E$ lines is an evidence that the class II masers in these objects are pumped by the radiation of hot dust rather than by that of UC HII-regions.
\end{abstract}

\section{Introduction}

Methanol masers were found in hundreds of Galactic star-forming regions. According to the classification by Menten (1991), all methanol masers can be divided into two classes, I and II. The strongest class I masers were found in the $7_{0}-6{ }_{1} A^{+}$and the $4_{-1}-3_{0} E$ transitions at 7 and $8 \mathrm{~mm}$, respectively. The weaker $5_{-1}-4_{0} E, 6_{-1}-5_{0} E$, and $8_{0}-7_{1} A^{+}$masers emit at shorter wavelengths, 3 and $2 \mathrm{~mm}$. The strongest class II masers, $2_{-1}-3_{0} E$ and $5_{1}-6_{0} A^{+}$are observed in the centimeter wave range, and the weaker $3_{0}-4_{1} A^{+}$and $J_{0}-J_{-1} E$ masers are observed at 3 and $2 \mathrm{~mm}$. Theoretical models of methanol excitation demonstrate that the class I masers are pumped by collisions, whereas the class II masers are pumped by external radiation (Sobolev et al. 1997). As a rule, methanol maser sources emit in several lines of the same class.

The dependence of maser intensity on wavelength, noted above, appears since both the degree of inversion and line opacities diminish with the decrease 

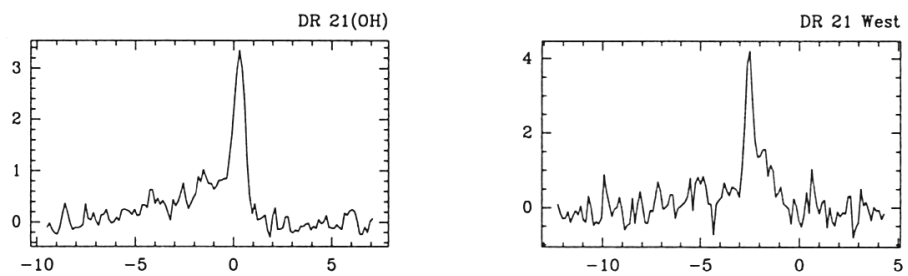

$229.8+230.0 \mathrm{GHz}$
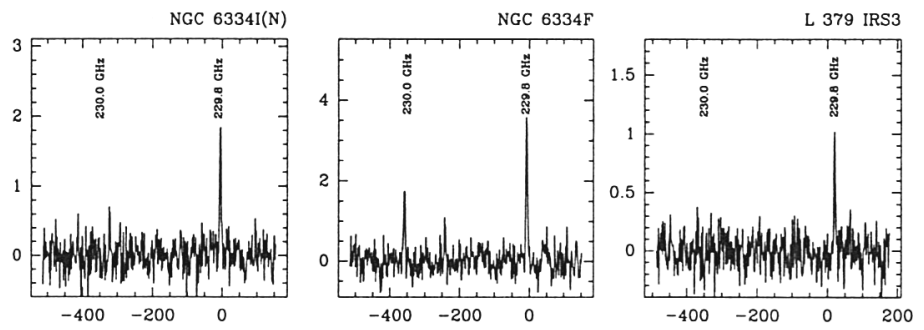

$108.9 \mathrm{GHz}$
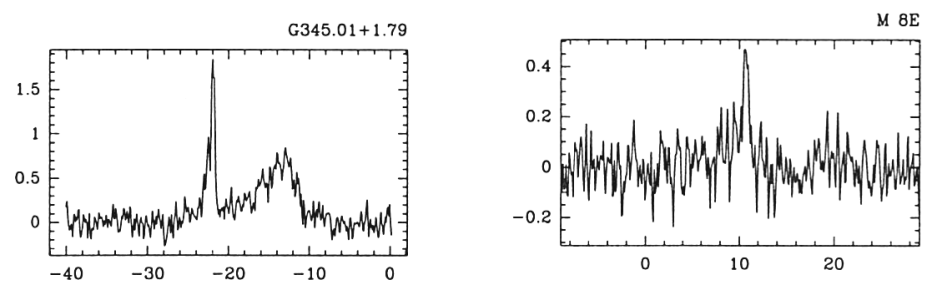

Figure 1. Spectra of maser and thermal emission at 229.8 and $108.9 \mathrm{GHz}$. x-axis, $V_{L S R} ; \mathrm{y}$-axis, $T_{R}^{*}$. $1 \mathrm{~K}$ of $T_{R}^{*}$ corresponds to $10.5 \mathrm{Jy}$ at $229 \mathrm{GHz}$, and to $6.3 \mathrm{Jy}$ at $108 \mathrm{GHz}$. DR 21(OH), DR 21 West, NGC $6334 \mathrm{I}(\mathrm{N})$, and L 379 IRS3 are class I masers, G345.01+1.79 and $\mathrm{M} 8 \mathrm{E}$ are class II masers. NGC $6334 \mathrm{~F}$ is a thermal source.

of wavelength. To determine the parameters of maser sources one must know the shortest wavelength at which the masers exist. Therefore we performed a search for the $8_{-1}-7_{0} E$ masers at $229.8 \mathrm{GHz}(1 \mathrm{~mm})$. No searches for methanol masers have been performed at so high frequencies before. The $8_{-1}-7_{0} E$ transition belongs to the class I, and hence the masers at $229.8 \mathrm{GHz}$ can be observed together with other masers of this class. The observations were carried out with the 30-m IRAM telescope. Besides the $8_{-1}-7_{0} E$ line, we observed the $0_{0}-1_{-1} E$ line at $108.9(3 \mathrm{~mm})$, the series of the $J_{1}-J_{0} E$ lines at $165 \mathrm{GHz}(2 \mathrm{~mm})$, and the $3_{-2}-4_{-1} E$ line at $230.0 \mathrm{GHz}(1 \mathrm{~mm})$.

\section{Results}

In the $8_{-1}-7_{0} E$ transition narrow maser features were found towards DR $21(\mathrm{OH})$ and DR 21 West (Fig. 1). Thus, for the first time methanol masers at $1 \mathrm{~mm}$ 
were found. Broad quasi-thermal lines were detected in 18 sources. We believe that at least in two of them, L 379IRS3 and NGC 6334I(N) these lines consist of blended narrow maser features, since other class I lines, detected in these sources are blends of several maser components (Slysh et al., 1997; Kogan and Slysh, 1998).

In the $0_{0}-1_{-1} E$ line, maser emission was found towards G345.01+1.79 and probably, towards $\mathrm{M} 8 \mathrm{E}$. In addition, 28 thermal sources were found in this line, including marginally detected galaxy IC 342 . Only thermal lines were found in the $3_{-2}-4_{-1} E$ and $J_{1}-J_{0} E$ transitions.

\section{Class I masers}

To determine the conditions, which are necessary to excite the $8_{-1}-7_{0} E$ masers, we calculated a number of LVG models with gas kinetic temperature 10-100 K, density $10^{4}-10^{8} \mathrm{~cm}^{-3}$, and methanol specific column density $2 \times 10^{13}$ to $2 \times 10^{17} \mathrm{~cm}^{-2} /(\mathrm{km} / \mathrm{s})$. Any external radiation, except the microwave background, was set equal to zero.

\begin{tabular}{l|rrrr}
\hline & \multicolumn{4}{|c}{ Flux density, Jy } \\
Transition & DR 21(OH) & DR 21 & West \\
& model & obs. & model & obs \\
\hline $4_{-1}-3_{0} E$ & 15.0 & 15 & 50.0 & 50 \\
$4_{0}-3_{1} E$ & 0.4 & $<0.7$ & 1.2 & $<2.8$ \\
$5_{-1}-4_{0} E$ & 37.8 & 135 & 82.0 & 100 \\
$6_{-1}-5_{0} E$ & 45.6 & 67 & 82.5 & 64 \\
$8_{-1}-7_{0} E$ & 32.7 & 25 & 40.0 & 34 \\
$9_{-1}-8_{-2} E$ & 0.003 & $<0.23$ & 0.004 & $<0.25$ \\
& & & & \\
$7_{0}-6_{1} A^{+}$ & 35.9 & 340 & 52.5 & 240 \\
$8_{0}-7_{1} A^{+}$ & 27.2 & 175 & 28.5 & 150 \\
\hline
\end{tabular}

Table 1. The observed and modelled flux densities in the class I lines. The model parameters: DR $21(\mathrm{OH}), T_{\text {kin }}=60 \mathrm{~K}, n_{\mathrm{H}_{2}}=5.6 \times 10^{4} \mathrm{~cm}^{-3}$, $N_{\mathrm{CH}_{3} \mathrm{OH}} / d V=2 \times 10^{16} \mathrm{~cm}^{-2} /(\mathrm{km} / \mathrm{s})$. DR 21 West, $T_{\text {kin }}=55, n_{\mathrm{H}_{2}}=$ $3.2 \times 10^{4} \mathrm{~cm}^{-3}, n_{\mathrm{CH}_{3} \mathrm{OH}} /(d V / d R)=2 \times 10^{16} \mathrm{~cm}^{-2} /(\mathrm{km} / \mathrm{s})$.

The modeling showed that the $8_{-1}-7_{0} E$ masers having intensities comparable to those of other class I masers can arise at gas temperature $30-50 \mathrm{~K}$, typical for the Galactic molecular clouds. Table 1 demonstrates the observed flux densities in various class I lines for DR $21(\mathrm{OH})$ and DR 21 West as well as flux densities in the best-fit models which were found using the $\chi^{2}$ criterion. None of the models represents well the observational data. This fact is probably related to the model simplifications, e.g., to the assumption that the same regions emit in all lines. Actually, maser regions may consist of several layers, and different maser lines may form in different layers. 


\section{Class II masers}

Class II maser emission was found only in the $0_{0}-1_{-1} E$ transition. Masers were detected towards G345.01+1.79, and possibly, towards M 8E.

The class II masers arise owing to a strong external radiation, which can be produced either by hot dust or by free-free transitions of electrons in HII-regions. We modelled the methanol excitation under the influence of the radio emission of HII-regions. A number of models accounting for the hot dust emission was calculated by Sobolev et al. (1997).

Slysh et al. (1995) detected the $J_{0}-J_{-1} E$ masers towards W $3(\mathrm{OH})$, G345.01+1.79, W 48, and Cep A. Our modelling of the class II masers pumping by the radio emission of HII-regions showed that this pumping always produce the $3_{-2}-4_{-1} E, J_{1}-J_{0} E$, and the $5_{-2}-6_{-1} E$ masers, comparable in intensity with the $J_{0}-J_{-1} E$ masers. However, the $3_{-2}-4_{-1} E$ and $J_{1}-J_{0} E$ masers were not found in this survey, and the $5_{-2}-6_{-1} E$ masers were not found by Slysh et al. (1997). At the same time, the models of methanol pumping by the emission of hot dust, published by Sobolev et al. (1997) predict that the intensity of the $J_{0}-J_{-1} E$ masers is larger than that of the $J_{1}-J_{0} E$ masers, and no strong maser emission arise in the $3_{-2}-4_{-1} E$ and $5_{-2}-6{ }_{-1} E$ lines, in a qualititative agreement with the observations.

Thus, the results of our observations, combined with the results by Slysh et al. $(1995,1997)$ mean that the hot dust emission must be taken into account in the modelling of class II methanol masers.

Our modelling shows that the $0_{0}-1_{-1} E$ transition is thermalized with the increase of either density or methanol content much earlier than any other class II line. This fact explains why the masers at $108.9 \mathrm{GHz}$ are so rare. The masers in G345.01+1.79 and M 8E are likely to be either the least dense class II masers, or their geometry (e.g., disk-like structure) provides an efficient photon escape.

Acknowledgments. The authors are grateful to the Pico Veleta observatory staff for the help during the observations. The work was done under a partial financial support from the International Scientific Foundation (grant No. MND300) and the Russian Foundation for Basic Research (grants No. 95-0205826 and 98-02-16916).

\section{References}

Kogan, L.R. \& Slysh, V.I. 1998, ApJ, 497, 800

Menten K.M., in: Publ. Astron. Soc. Pac., Skylines, Proc. 3rd Haystack Observatory Meeting, eds. A.D. Haschick, P.T.P. Ho, 119

Slysh, V.I., Kalenskii, S.V. \& Val'tts I.E. 1995, ApJ, 42, 668

Slysh, V.I., Kalenskii, S.V., Val'tts, I.E., \& Golubev, V.V. 1997, ApJ, 478, L37 Sobolev, A.M., Cragg, D., \& Godfrey, P.D. 1997, MNRAS, 288, L39

Val'tts, I.E., Ellingsen, S.P., Slysh, V.I., Kalenskii, S.V., Otrupcek, R., \& Voronkov, M.A. 1999, MNRAS, 310, 1077 